\title{
High-speed 3D imaging using a chaos lidar system
}

\author{
Hsin-Lin Ho, Jun-Da Chen, Ching-An Yang, Chia-Chi Liu, Cheng-Ting Lee, Yu-Hsiang Lai, and Fan-Yi Lin ${ }^{\mathrm{a}}$ (D) \\ Institute of Photonics Technologies, Department of Electrical Engineering, National Tsing Hua University, Hsinchu 300, \\ Taiwan
}

Received 8 August 2021 / Accepted 16 December 2021

(C) The Author(s) 2022, corrected publication 2023

\begin{abstract}
We characterize a new chaos lidar system configuration and demonstrate its capability for highspeed 3D imaging. Compared with a homodyned scheme employing single-element avalanche photodetectors (APDs), the proposed scheme utilizes a fiber Bragg grating and quadrant APDs to substantially increase the system throughput, frame rate, and field-of-view. By quantitatively analyzing the signal-tonoise ratio, peak-to-standard deviation of the sidelobe level, precision, and detection probability, we show that the proposed scheme has better detection performance suitable for practical applications. To show the feasibility of the chaos lidar system, while under the constrain of eye-safe regulation, we demonstrate high-speed 3D imaging with indoor and outdoor scenes at a throughput of $100 \mathrm{kHz}$, a frame rate of $10 \mathrm{~Hz}$, and a FOV of $24.5^{\circ} \times 11.5^{\circ}$ for the first time.
\end{abstract}

\section{Introduction}

In recent years, lidars have been widely used in sensing applications such as autonomous vehicles, augmented reality and virtual reality (AR/VR), simultaneous localization and mapping, and industrial automation [1-4]. Lidars having high throughput and fast beam scanning capability can acquire 3D images of the targets or surroundings with high precision up to a relatively long range $[5,6]$. Conventional pulsed lidars measure the time-of-flight (TOF) between the transmitted and the backscattered light from the target to obtain the range information $[7,8]$. They have the advantages of long-range and fast detection by emitting repetitive short pulses with high peak power $[9,10]$. However, with the unspecific waveforms emitted, the pulsed lidars are inevitably vulnerable to jamming and can easily be interfered with by other lidars or stray and ambient light in the environment $[11,12]$.

Random-modulation continuous-wave (RM-CW) lidars [13-15] and chaos lidars [16-19] carrying specific waveforms have been proposed to mitigate the issue of interference and jamming. However, although the concepts of these correlation-based lidars were proved, emitting the light in a CW form limits both their peak power and throughput (pixel rate) and makes them unfavorable for high-speed 3D imaging.

Generations of chaos-modulated pulses have been proposed in various schemes for chaos lidar applications to improve the detection capability [20-22]. In 2018 , the authors reported an eye-safe pulsed 3D chaos lidar system employing self-homodyning and time gat-

\footnotetext{
a e-mail: fylin@ee.nthu.edu.tw (corresponding author)
}

ing to generate chaos modulated pulses to increase peak power and signal-to-noise ratio (SNR) [23]. Snapshot $3 \mathrm{D}$ images with sub-centimeter precision have been successfully demonstrated using a chaos lidar for the first time. However, the field-of-view (FOV) was less than $2^{\circ}$ due to the small active area of the detector and the still inefficient pulses generated. Recently, improvement has been made by employing a pulsed master oscillation power amplifier (MOPA) scheme to enhance system's peak-to-standard deviation of the sidelobe level ( $\left.\mathrm{PSL}_{\mathrm{std}}\right)$. Consequently, throughput increased from 1 to $6 \mathrm{kHz}$ and FOV doubled to above $4^{\circ}$ [24]. Despite these advancements, the frame rate achieved was still less than $1 \mathrm{~Hz}$.

In this study, we employ a fiber Bragg grating (FBG) in the chaos light source module to reduce the chaos signal's bandwidth to better match detector's bandwidth and increase energy efficiency. Hence, high-speed 3D imaging with a higher frame rate and larger FOV can be realized for more versatile applications. We replace the lossy acousto-optic modulator (AOM) with a gainmodulated booster optical amplifier (BOA) to transform the CW chaos oscillations into chaos-modulated pulses for higher peak power and better SNR. In the optical transceiver module, we operate the microelectromechanical systems (MEMS) mirror in its resonant mode to accelerate the line rate and increase the frame rate. We replace the single-element avalanche photodetectors (APDs) with quadrant APDs to increase the effective detection area. Also, by optimizing the receiver optics with multiple lenses having defocused coupling to the APD, we maximize the system's FOV. With this new configuration while under the constrain of eye-safe regulation [25], we achieve high-speed 3D imaging with 
a throughput of $100 \mathrm{kHz}$, a frame rate of $10 \mathrm{~Hz}$, and a FOV of $24.5^{\circ} \times 11.5^{\circ}$ in a chaos lidar for the first time.

\section{Experimental setup of 3D pulsed chaos lidar}

Figure 1 shows a 3D pulsed chaos lidar system schematic, mainly comprising a chaos light source, an optical transceiver, and a signal acquisition and processing module. The chaos light source module generates CW chaos oscillations with a 1550-nm single-mode semiconductor laser (Shengshi Optical SBF-D55W2$111 \mathrm{PMS}$ ) subject to optical feedback with a normalized feedback strength of 0.017 and a time delay of 65 ns [23]. An optical isolator (GIP PMOI151BL120001A) with an extinction ratio of $20 \mathrm{~dB}$ is placed after the chaos laser to prevent any unwanted feedback. Since the bandwidth of the chaos (typically several $\mathrm{GHz}^{-}$ tens of $\mathrm{GHz}$ ) is much broader than the detection bandwidth (APDs typically have bandwidths of hundreds of $\mathrm{MHz}$ ), we use a FBG (iXblue Photonics IXC-FBGPS-1550-1-ATH-PM-C, linewidth $8 \mathrm{pm}$ ) to reduce the chaos signal's bandwidth and enhance energy efficiency. Moreover, to transform the $\mathrm{CW}$ chaos oscillations into chaos-modulated pulses for higher peak power, we use a gain-modulated BOA (Thorlabs BOA1004PXS) driven by a pulse driver (AeroDIODE CCS-std) to enhance the SNR. A band-pass filter (BPF, 0.3-nm linewidth) is used to reduce the amplified spontaneous emission noise from the BOA. Pulses are amplified with an erbiumdoped fiber amplifier (EDFA, GIP CGB1E3128001A) in a MOPA scheme to boost the peak power further. Before sending the pulses to the EDFA, we use a
30:70 fiber coupler to split the light and detect the transmitted chaos-modulated pulses with a quadrant APD (Idealphotonics QPD-1000) as the reference waveform. Coupled with a laboratory-made transimpedance amplifier (TIA), the detector has a 3-dB bandwidth of $250 \mathrm{MHz}$.

In the optical transceiver module, the amplified chaos-modulated pulse is coupled out to the free-space through a collimator and then scanned by a two-axis MEMS mirror (Mirrorcle, S6244) to acquire the 3D images. We use a combination of two 2-inches Fresnel lenses and a 4-mm half-ball lens in front of an APD identical to the one used for the reference waveform to collect the backscattered light from the target and detect the received chaos-modulated pulses as the signal waveform.

In the signal acquisition and processing module, the oscilloscope (Tektronix, MSO58) simultaneously acquires the reference and signal waveforms at a sampling rate of $1.25 \mathrm{GHz}$. The range of the target is obtained by calculating the lag time of the crosscorrelation peak between the reference and signal using a personal computer. We use a second-order Chebyshev high-pass digital filter with a cutoff frequency of $1 \mathrm{MHz}$ to remove the square-wave modulation in the waveforms that define the width of the pulses. The span of the correlation window is $100 \mathrm{~ns}$, the same as the pulsewidth. To improve the precision in ranging, we apply the Spline interpolation in MATLAB to determine the time of the correlation peak more precisely $[26,27]$. The MEMS controller sends a trigger signal to the function generator (FG, Agilent 81150A). Further, it sends triggers to the pulse driver and the oscilloscope for all modules to synchronize.
Fig. 1 Schematic setup of a $3 \mathrm{D}$ pulsed chaos lidar system. Chaos laser: a single-mode semiconductor laser subject to optical feedback; $F B G$ fiber Bragg grating; $B O A$ booster optical amplifier; $B P F$ band-pass filter; $F C$ fiber coupler; $V A$ variable optical attenuator; $A P D$ avalanche photodetector; $F G$ function generator; $E D F A$ erbium-doped fiber amplifier; $M E M S$ microelectromechanical systems; $O S C$ oscilloscope; $P C$ personal computer







Fig. 2 a Optical spectra, b power spectra, and $\mathbf{c}$ waveforms of the chaos signals generated by the FBG scheme (red) and the homodyned scheme (blue). The black curve in (b) is the noise spectrum of the APD used for the measurement

\section{Characteristics of chaos light source}

Figure 2a, b shows the optical and power spectra of the chaos signals generated by the FBG scheme (red) and the homodyned scheme [23] (blue), respectively. With the same amount of optical power received by the signal APD, the signal generated by the FBG scheme has a narrower linewidth governed by the FBG and a modulation power about $8 \mathrm{~dB}$ higher than that generated by the homodyned scheme. After transforming the $\mathrm{CW}$ chaos oscillations into chaos-modulated pulses by the BOA, as the waveforms shown in Fig. 2c, the modulation of the FBG scheme is much larger, and the corresponding SNR is about 2.5 times (4 dB) higher than that of the homodyned scheme. SNR is defined as the ratio between the standard deviation of the chaos modulation amplitude to the standard deviation of the noise.

Figure 3a-d shows the respective SNR, $\mathrm{PSL}_{\text {std }}$, precision, and detection probability of the FBG and homodyned schemes obtained at various peak powers of the received signal (electrical signal acquired by the ADC) to quantify their detection performance. Kodak white card (90\% reflectance, diffuse reflection) placed at a distance of $1 \mathrm{~m}$ from the system is used as a standardized target. At each peak power, for comparison, the optical power received by the signal APD (also the reference APD) in both schemes are kept the same. Here the optical power is adjusted so that, at the highest peak power of $4 \mathrm{dBm}$, the magnitude of the received waveform for the FBG scheme as shown in Fig. 2c is just below the saturation voltage of the TIA $(0.6 \mathrm{~V})$ to prevent saturation. The averages and standard deviations of SNR and $\mathrm{PSL}_{\text {std }}$ (defined as the ratio between the cross-correlation peak and three times the standard deviation of the noise floor in the correlation trace) from 100 consecutive measurements are shown.

As can be seen in Fig. 3a, b, the SNR and the corresponding $\mathrm{PSL}_{\mathrm{std}}$ of both schemes are linearly proportional to the peak power of the received signal. Due to larger modulation, for the same peak power, the SNR and PSL $_{\text {std }}$ of the FBG scheme are about $4 \mathrm{~dB}$ higher than that of the homodyned scheme. For specific power, the $\mathrm{PSL}_{\mathrm{std}}$ is about $3 \mathrm{~dB}$ higher than SNR in both schemes due to the noise filtering in the crosscorrelation process when calculating the $\mathrm{PSL}_{\mathrm{std}}$.

For similar signal bandwidth and pulsewidth, the precision of the chaos lidar system is mainly determined by the SNR and the corresponding $\mathrm{PSL}_{\text {std }}[24,28,29]$. Here, precision is defined as the standard deviation of 100 consecutive range measurements from a fixed target. The precision of both schemes decreases as the peak power increases, as shown in Fig. 3c. For the same power, the precision of the FBG scheme is substantially better (lower) than that of the homodyned scheme due to higher SNR. Note that, while the detection area of the quadrant APD used in this study (1 mm diameter) is larger than the single-element APD used in the previous setup (Ref. [24], $0.2 \mathrm{~mm}$ diameter), the 3 - $\mathrm{dB}$ bandwidth of the detector reduces from $400 \mathrm{MHz}$ to $250 \mathrm{MHz}$. Hence, as a trade-off for extending FOV, the optimum precisions shown here for both schemes are not as good as that obtained in the previous study [24]. Reference [24] presents a detailed analysis of the precision of a chaos lidar system.

In Fig. 3d, we show the detection probability of the chaos lidar system at various peak powers of the received signal for the FBG and homodyned schemes. Here, the detection probability is defined as the ratio of the number of valid detection over total detection. A valid detection is accounted for when the detection has a range error less than half of the sampling size. In this study, 100 consecutive detections are performed at each power and the sampling size is $12 \mathrm{~cm}$ associated with the sampling rate of $1.25 \mathrm{GHz}$. As can be seen, to have a detection probability above $90 \%$, the lowest peak power required in the FBG scheme is about $9 \mathrm{~dB}$ lower than the homodyned scheme. From the above, by better matching the chaos signal's bandwidth to the detector's, we show that the FBG scheme has advantages over the homodyned scheme for chaos lidar detection.

\section{Enhancing the detection FOV}

$\mathrm{PSL}_{\text {std }}$ is measured at different scanning angles (shown in Fig. 4a) to quantify the detection FOV of the chaos 
Fig. 3 a SNR, b $\mathrm{PSL}_{\mathrm{std}}$, c precision, and $\mathbf{d}$ detection probability of the FBG and homodyned schemes obtained at various peak powers of the received signal. The SNR and $\mathrm{PSL}_{\text {std }}$ shown are the averages and standard deviations from 100 consecutive measurements. The red and blue lines are the curve-fittings. The black dashed line indicates a detection probability of $90 \%$
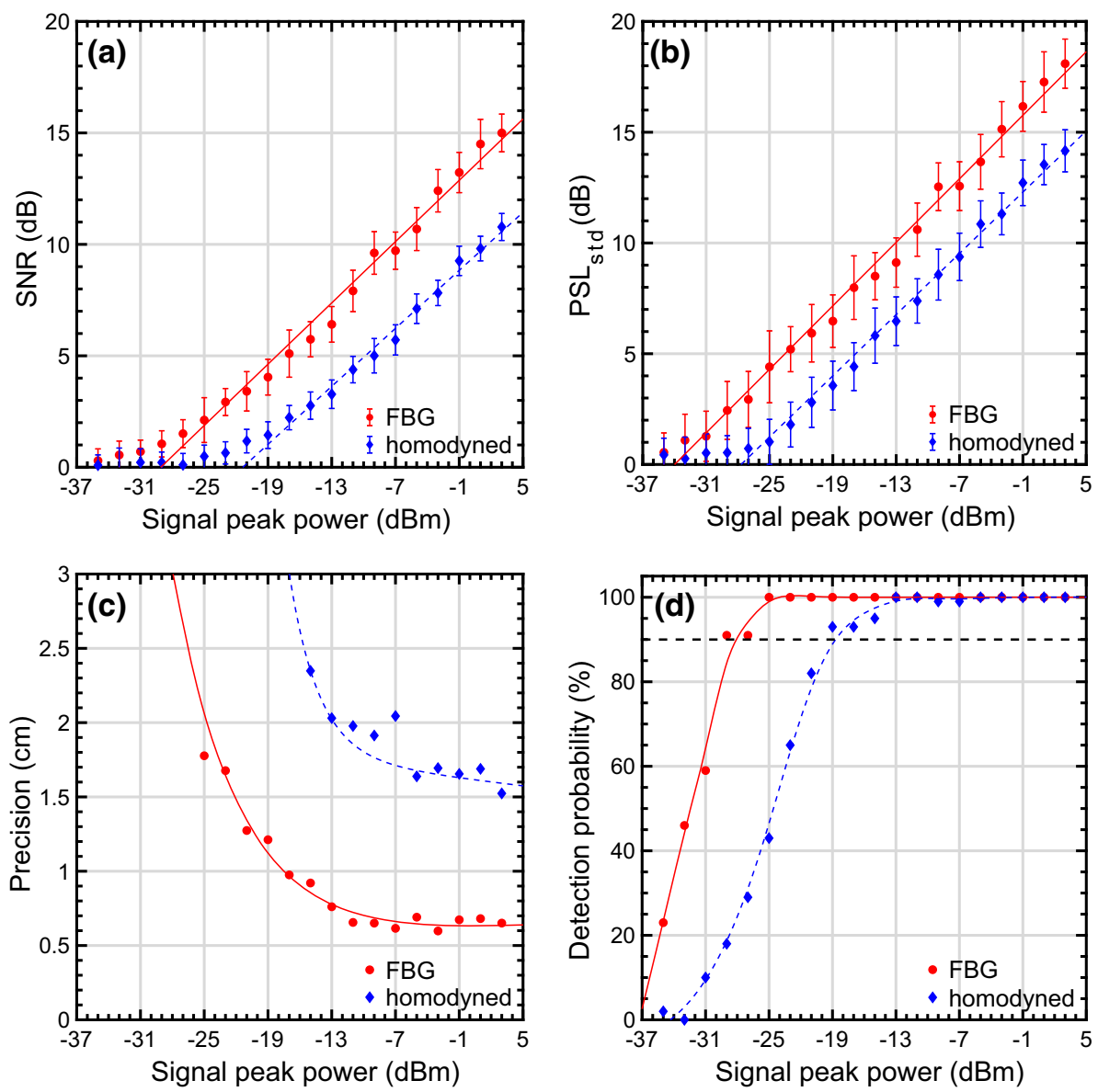



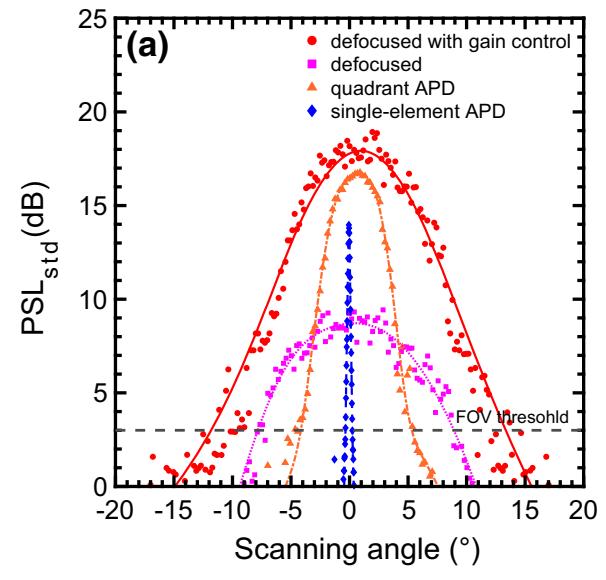

Fig. 4 a PSL $_{\text {std }}$ obtained at different scanning angles acquired from the single-element APD, quadrant APD, quadrant APD with defocusing, and defocused quadrant APD with gain control, respectively. The detection FOV is

lidar system adopting the FBG scheme. The same standardized target is placed at $5 \mathrm{~m}$, and the EDFA output is $10 \mathrm{dBm}$. Here, detection FOV is the angular span that the detector can receive backscattered light and has $\mathrm{PSL}_{\text {std }}$ above $3 \mathrm{~dB}$. Due to the larger detection area and collecting more light, the $\mathrm{PSL}_{\text {std }}$ acquired by the quadrant APD is generally greater than that using the single-element APD. As a result, the FOV obtained



defined to be the angular span that has $\mathrm{PSL}_{\text {std }}$ above $3 \mathrm{~dB}$ (black dashed line). b FOV at different detection ranges with the quadrant APD under defocusing and gain control

by the quadrant APD is $9.4^{\circ}$ while that obtained by the single-element APD is merely $0.6^{\circ}$.

To distribute the energy more evenly and maximize the FOV, we intentionally defocus the received light by placing the detector $10 \mathrm{~mm}$ closer to the Fresnel lens from its focal point. Hence, the power and $\mathrm{PSL}_{\text {std }}$ are redistributed to broader scanning angles, and the FOV is almost doubled at $16.7^{\circ}$. Nevertheless, while 
defocusing enhances the FOV, it comes with the tradeoff of reducing the $\mathrm{PSL}_{\text {std }}$ at the central region of the detection.

To further maximize the FOV, we apply gain control to adjust the emitting power, maximize the magnitude of the received waveform, and let it be just below the saturation voltage of the TIA. PSL std $_{\text {increases signifi- }}$ cantly with an increase in the emitting power from 10 to $19 \mathrm{dBm}$. It also enhances the FOV to $25.2^{\circ}$.

Figure 4b shows the FOV obtained with the setup facilitating the quadrant APD for a larger detection area, defocusing to redistribute the energy more evenly, and gain control to maximize the emitting power and $\mathrm{PSL}_{\text {std }}$ at different detection ranges. In the range between 5 and $20 \mathrm{~m}$, FOVs between 25.2 and $31.5^{\circ}$ are achieved making it versatile for many potential applications.

\section{Demonstration of high-speed 3D imaging}

To demonstrate high-speed 3D imaging using the chaos lidar, we operate the pulsed MOPA at an average output power of $27 \mathrm{dBm}$ with a pulse repetition frequency of $100 \mathrm{kHz}$ and a pulsewidth of $100 \mathrm{~ns}$. By employing the two-axis MEMS mirror scanning at a resonant line rate of $1 \mathrm{kHz}$ in one axis and a linear scan of $10 \mathrm{~Hz}$ in the other, point clouds and their corresponding 3D rendering with a FOV of $24.5^{\circ} \times 11.5^{\circ}$, a resolution of 100 $\times 100$ pixels, a throughput of $100 \mathrm{kHz}$, and a frame rate of $10 \mathrm{~Hz}$ can be acquired. We ensure the average optical power and pulse energy that may enter the human pupil to be less than $10 \mathrm{dBm}$ and $8 \mathrm{~mJ}$ to comply with the eye-safe regulation [25]. For better demonstration, noise filtering is used at the post-processing to remove obvious outliers in the point cloud.

Note that, the FOV in the axis operated in the resonant mode is much larger than the one in the linear mode. Differ from the detection FOV shown in Fig. 4 that is governed by the size of the detector and the optimization of coupling, the FOV here is limited by the maximum mechanical angle the MEMS mirror can scan. While it can be extended with the use of wideangle lenses, the spot size of the emitting beam may be increased accordingly.

Figure $5 \mathrm{a}-\mathrm{c}$ shows a video of a person dribbling and passing a basketball and the front and bird's-eye views of the corresponding 3D rendering obtained from the acquired point cloud. The target is about $9 \mathrm{~m}$ away and moving towards the lidar. Each pixel is shown with its corresponding $\mathrm{PSL}_{\text {std }}$ value indicated by its color. As shown, at a frame rate of $10 \mathrm{~Hz}$, the posture, movement, and trajectory of the ball are clearly recorded. [Please see website for animations]

Figure $6 \mathrm{a}-\mathrm{c}$ demonstrates a video of a person signaling a moving vehicle and the front and bird's-eye views of the corresponding 3D rendering obtained from the acquired point cloud. The lidar system is positioned outdoor directly under the sunlight without using any infrared filter. The vehicle is about $11 \mathrm{~m}$ moving away from the lidar. As shown, at a frame rate of $10 \mathrm{~Hz}$, the

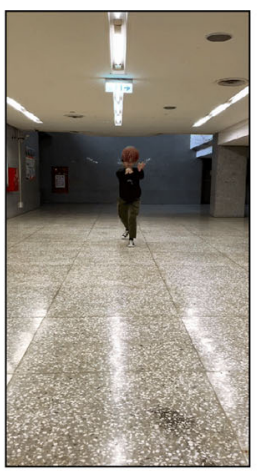

(a)

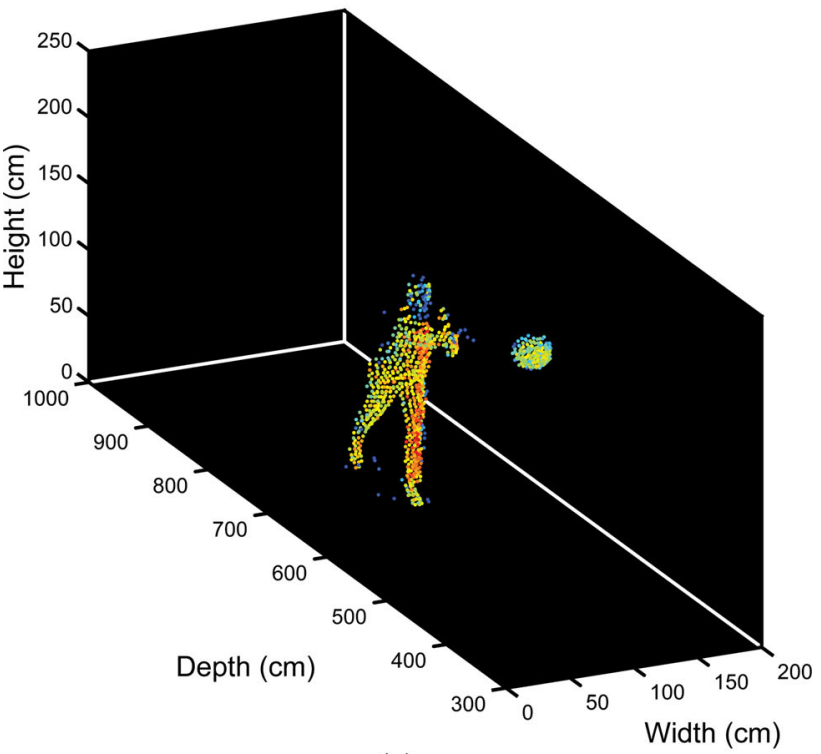

(c)

Fig. 5 a Photo of a person dribbling and passing a basketball $\mathbf{b}$ front view $\mathbf{c}$ bird's eye view of the corresponding 3D rendering obtained from the acquired point cloud. Each pixel is shown with its corresponding PSL std value indicated by its color [Please see website for animations]. This image is a moving image and can be viewed via the link given in the electronic supplementary material section

vehicle's details including the contour, windows, and wheels are clearly depicted [Please see website for animations]. Without any significant effect of the ambient light due to its specific waveform and correlationbased nature, we show the capability and potential of the chaos lidar in real-world 3D applications.

\section{Conclusions}

In summary, we study the characteristics of a chaos lidar system utilizing an FBG and quadrant APDs for better detection performance. Compared with a homodyned scheme using single-element APDs, the proposed scheme is about $4 \mathrm{~dB}$ higher in SNR and PSL $\mathrm{PL}_{\text {std }}$ and therefore has better precision and detection probability. By further employing defocusing and gain control, the 
Fig. 6 a Photo of a person signaling a moving vehicle outdoor $\mathbf{b}$ front view c bird's eye view of the corresponding 3D rendering obtained from the acquired point cloud. Each pixel is shown with its corresponding $\mathrm{PSL}_{\text {std }}$ value indicated by its color [Please see website for animations]. This image is a moving image and can be viewed via the link given in the electronic supplementary material section



(a)

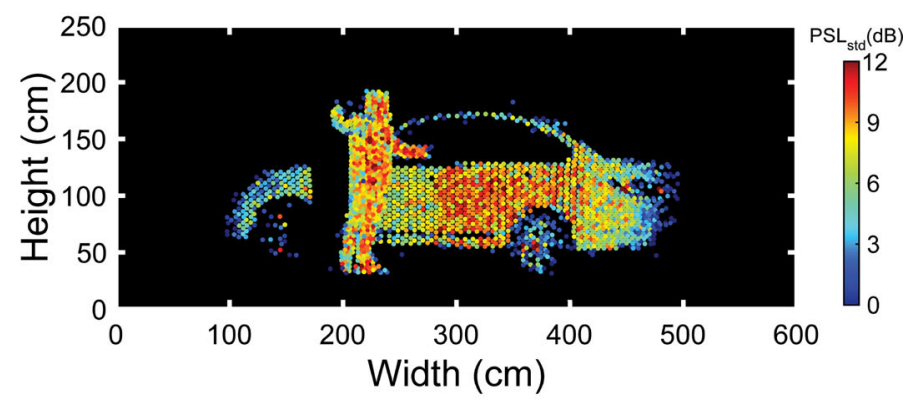

(b)

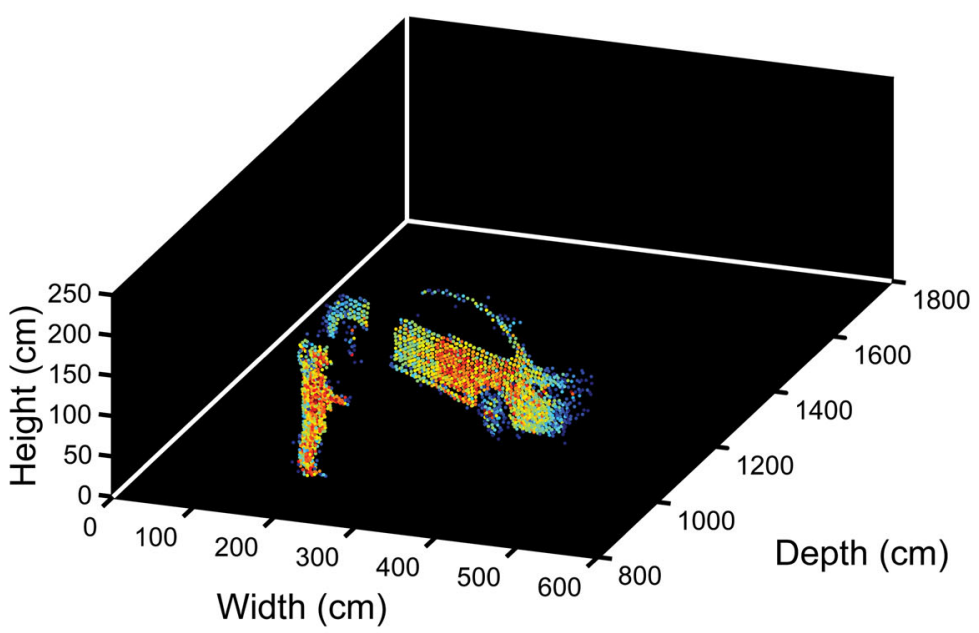

(c)

proposed scheme extends the detection FOV to more than $25.2^{\circ}$ in the range between 5 to $20 \mathrm{~m}$. With these significant improvements, we demonstrate high-speed $3 \mathrm{D}$ imaging at a throughput of $100 \mathrm{kHz}$, a frame rate of $10 \mathrm{~Hz}$, and a FOV of $24.5^{\circ} \times 11.5^{\circ}$ using a chaos lidar system for the first time. With future advancements in fabricating APD arrays with more elements to further increase the effective detection area and/or bandwidth, chaos lidar systems with even better precision and FOV are expected.

Supplementary information The online version contains supplementary material available at https://doi.org/ 10.1140/epjs/s11734-021-00410-8.

Open Access This article is licensed under a Creative Commons Attribution 4.0 International License, which permits use, sharing, adaptation, distribution and reproduction in any medium or format, as long as you give appropriate credit to the original author(s) and the source, provide a link to the Creative Commons licence, and indicate if changes were made. The images or other third party material in this article are included in the article's Creative Commons licence, unless indicated otherwise in a credit line to the material. If material is not included in the article's Creative Commons licence and your intended use is not permitted by statutory regulation or exceeds the permitted use, you will need to obtain permission directly from the copyright holder. To view a copy of this licence, visit http://creativecomm ons.org/licenses/by/4.0/.

\section{References}

1. B. Schwarz, Nat. Photonics 4, 429-430 (2010)

2. Q. Li, L. Chen, M. Li, S.L. Shaw, A. Nuchter, IEEE Trans. Veh. Technol. 63(2), 540-555 (2014)

3. J. McCormack, J. Prine, B. Trowbridge, A. C. Rodriguez, R. Integlia, Proceedings of IEEE Games Entertainment Media Conference (GEM), pp. 1-5 (2015)

4. F. Moosmann, C. Stiller, Proceedings of IEEE Intelligent Vehicles Symposium (IV), pp. 393-398 (2011)

5. R.H. Rasshofer, K. Gresser, Adv. Radio Sci. 3, 205-209 (2005)

6. Y. Lin, J. Hyyppa, A. Jaakkola, J. Yang, Proceedings of IEEE Geoscience and Remote Sensing Letters, p. 426430 (2011)

7. M.C. Amann, T. Bosch, M. Lescure, R. Myllyla, M. Rioux, Opt. Eng. 40(1), 10-19 (2001)

8. R. Horaud, M. Hansard, G. Evangelidis, C. Menier, Mach. Vis. Appl. 27(7), 1005-1020 (2016)

9. M.U. Piracha, D. Nguyen, D. Mandridis, T. Yilmaz, I. Ozdur, S. Ozharar, P.J. Delfyett, Opt. Express 18, 7184-7189 (2010)

10. J.D. Spinhirne, IEEE Trans. Geosci. Remote Sens. 31(1), 48-55 (1993)

11. G. Kim, J. Eom, Y. Park, Proceedings of IEEE Intelligent Vehicles Symposium (IV), pp. 437-442 (2015)

12. J. Petit, B. Stottelaar, M. Feiri, F. Kargl, Proceedings of Black Hat Europe (2015)

13. X. Ai, R. Nock, N. Dahnoun, J.G. Rarity, Appl. Opt. 50(22), 4478-4488 (2011) 
Eur. Phys. J. Spec. Top.

14. R. Matthey, V. Mitev, Opt. Lasers Eng. 43, 557-571 (2005)

15. Z. Yang, C. Li, M. Yu, F. Chen, T. Wu, J. Appl. Remote. Sens. 9, 1-14 (2015)

16. F.Y. Lin, J.M. Liu, IEEE J. Sel. Top. Quantum Electron. 10, 991-997 (2004)

17. W.T. Wu, Y.H. Liao, F.Y. Lin, Opt. Express 18, 26155$26162(2010)$

18. C.H. Cheng, Y.C. Chen, F.Y. Lin, IEEE Photon. J. 8(1), 1-14 (2016)

19. F.Y. Lin, J.M. Liu, IEEE J. Quantum Electron. 40(6), 815-820 (2004)

20. H.L. Tsay, C.Y. Wang, J.D. Chen, F.Y. Lin, Opt. Express 28, 24037-24046 (2020)

21. C.Y. Chen, C.H. Cheng, D.K. Pan, F.Y. Lin, Opt. Express 26, 20851-20860 (2018)

22. K.T. Ting, F.Y. Lin, Opt. Express 26, 24294-24306 (2018)
23. C.H. Cheng, Y.C. Chen, J.D. Chen, D.K. Pan, K.T. Ting, F.Y. Lin, Opt. Express 26(9), 12230-12241 (2018)

24. J.D. Chen, H.L. Ho, H.L. Tsay, Y.L. Lee, C.A. Yang, K.W. Wu, J.L. Sun, D.J. Tsai, F.Y. Lin, Opt. Express 29(17), 27871-27881 (2021)

25. International Electrotechnical Commission (IEC), Safety of Laser Products. Part 1: Equipment Classification, Requirements and User's Guide, Standard IEC 60825-1:2001 (International Electrotechnical Commission, 2001), pp. 20-40

26. C. de Boor, A Practical Guide to Splines (Springer, New York, 1978)

27. G. Wahba, SIAM J. Sci. Stat. Comput. 2, 5-16 (1981)

28. L. Svilainis, K. Lukoseviciute, V. Dumbrava, A. Chaziachmetovas, Measurement 46, 3950-3958 (2013)

29. L. Svilainis, IEEE Trans. Ultrason. Ferroelectr. Control 66(11), 1691-1698 (2019) 